\title{
Clinical Trials Corner: A New Look at Cytoreductive Nephrectomy
}

Received 10 April 2021

Accepted 13 April 2021

Pre-press 19 May 2021

Published 16 June 2021

Dear Readers,

The Clinical Trials Corner of Kidney Cancer highlights planned or ongoing high-impact studies in renal cell carcinoma (RCC). In this issue, we highlight the PROBE trial, an important study evaluating the role of cytoreductive nephrectomy in the modern era of immunotherapy-based first-line systemic treatment.

In the future, if you feel that you would like to draw attention to a specific trial, please feel free to email us at mbparikh@ucdavis.edu orkca@iospress.com.

Sincerely,

Mamta Parikh, MD, MS

Associate Editor, Kidney Cancer

Assistant Professor, University of California Davis School of Medicine

Department of Internal Medicine

Division of Hematology Oncology

Sacramento, CA, USA

\section{A Phase III Trial of Immunotherapy-Based Combination Therapy With or Without Cytoreductive Nephrectomy for Metastatic Renal Cell Carcinoma (PROBE Trial).}

Status: Recruiting

Clinicaltrials.gov identifier: NCT04510597

Sponsor: Southwest Oncology Group

Enrollment: 364

Rationale: The benefit of cytoreductive nephrectomy $(\mathrm{CN})$ had previously been established among fit patients with metastatic renal cell carcinoma. Subsequently, however, systemic therapy for mRCC evolved significantly. During the era of anti-angiogenesis therapy as first-line treatment, the CARMENA study supported the deferral of $\mathrm{CN}$ in patients receiving sunitinib who had intermediate or poor risk disease. However, subsequently, treatment of $\mathrm{mRCC}$ has further evolved, and with the new paradigm of immunotherapy-based combination treatment in the first-line setting, the role of cytoreductive nephrectomy remains in question.

Study Design: This Phase III randomized trial enrolls patients with histologically proven clear cell or nonclear cell RCC. Patients may be treatment naïve or have initiated systemic therapy in the locally advanced or 
metastatic setting up to 12 weeks prior to registration as long as scans documented metastatic disease prior. Patients with symptomatic brain metastases, solitary kidney, or kidney transplantation are excluded. Patients without progression on combination immunotherapy-based systemic therapy at 12 weeks will be randomized to continue on systemic immunotherapy or to undergo cytoreductive nephrectomy while continuing on systemic immunotherapy.

Endpoints: The primary endpoint of this trial is comparison of overall survival between patients receiving immune checkpoint inhibitor-based combination therapy alone versus those who receive immune checkpoint inhibitor-based combination therapy and cytoreductive nephrectomy. The secondary outcomes include assessing complications of nephrectomy and post-randomization drug toxicities, objective response rate in metastatic sites between arms, and assessing change in diameter of primary tumor at week 12 disease assessment.

Comments: For over 50 years, spontaneous regression of sites of metastases have been reported in patients with $\mathrm{mRCC}$ after cytoreductive nephrectomy. In the era of interferon alfa- $2 b$ therapy, the benefit of nephrectomy was studied and shown to confer overall survival benefit in fit patients in Phase III SWOG and EORTC trials. With the advent of antiangiogenesis therapy for $\mathrm{mRCC}$, clinical trials sought to determine whether cytoreductive nephrectomy prior to initiation of systemic therapy remained of benefit. These trials proved to be challenging to accrue. Phase III CARMENA results argued that deferred nephrectomy was the preferred approach, but results may have been complicated by patient selection overrepresenting those with poor risk disease. Ultimately, though, the relevance of this study was short-lived in that systemic therapy has dramatically evolved for mRCC in recent years with the incorporation of immune checkpoint inhibitor therapy in front-line treatment, again raising the question of whether cytoreductive nephrectomy might benefit patients with mRCC. The SWOG PROBE trial is designed pragmatically to allow for a variety of first-line immune checkpoint inhibitor therapybased combinations and to allow for patients to have already initiated systemic therapy prior to enrollment. Patients in this study will not have upfront cytoreductive nephrectomy, but rather will be evaluated for benefit of cytoreductive nephrectomy after 12 weeks of systemic therapy. In addition to addressing the role of cytoreductive nephrectomy in treatment-naïve patients with $\mathrm{mRCC}$ who receive immune checkpoint inhibitor therapy, the study may shed further light on the impact of immunotherapy on the tumor microenvironment given that cytoreductive nephrectomy specimens will be collected in patients randomized to undergo surgery.

\section{CONFLICT OF INTEREST}

Mamta Parikh

Consultant: Janssen, Seagen, Oncocyte 